\title{
Development of a Cost-effective Xeno-free Conditioned Medium Containing Human Basic Fibroblast Growth Factor for the Expansion of Human Mesenchymal Stem Cell
}

\author{
Sung $\mathrm{J}^{1,2}$, Wu KC ${ }^{1,2}$, Choi MC ${ }^{1,2}$, Ma CHY ${ }^{1}$, Lai ATL ${ }^{1}$, Lin $\mathrm{J}^{1}$ and Kwong $\mathrm{KWY}^{1,2 *}$ \\ ${ }^{1}$ Research Department, DreamTec Research Limited, Kowloon, Hong Kong, P.R. China \\ ${ }^{2}$ Cytogenesis Corporation Limited Kowloon, Hong Kong, P.R. China
}

\begin{abstract}
Stem cells are a group of undifferentiated cells capable of regenerating somatic cells through cell division and differentiation. Among the lineage of stem cells, human mesenchymal stem cells (hMSCs) are adult stem cells that can be isolated from human tissues such as bone marrow, adipose tissues and amniotic fluids. Due to the ability of high differentiability into multiple lineages of different cell types, it is highly valuable in regenerative medicine. However, low consistent maintenance of differentiability and potency of stem cells, as well as expensive cultivation of stem cells impede the research and application of hMSCs in current medical fields. Hence, it is urging to find a more defined, low cost culture media in expansion of hMSCs without reducing its differentiability and potency. In this study, we demonstrated a well-defined xeno-free conditioned medium containing human basic fibroblast growth factor (FGF2) for hMSCs cultivation. Our results showed enhanced proliferation activity and successful maintenance of the elongated and spiral morphologies of hMSCs cultured in our conditioned medium supplemented with $100 \mathrm{ng} / \mathrm{mL}$ FGF2. More importantly, the undifferentiability of hMSCs was also validated by FACS, microscopy, qPCR and Western Blotting. We believe the present fine- tuned growth medium could be utilized for mass production of hMSCs.
\end{abstract}

Keywords: Mesenchymal Stem Cells (MSCs) • hMSCs • Expansion • Regenerative Medicine • Basic Fibroblast Growth Factor (FGF2)

\section{Introduction}

Due to its high accessibility, proliferation potential, inherent immunomodulatory, reparative properties and multiple differentiation capability, human mesenchymal stem cells (hMSCs) have been widely used as a reliable cell source for stem cell therapy, tissue engineering, drug discovery and disease modeling [1-9]. hMSCs are readily to be obtained conveniently and directly from bone marrow, adipose tissue, amniotic fluid (AF), umbilical cord and placenta for the seed culture of cell expansion $[1,10]$. While the potential of hMSCs has been confirmed by many researchers for therapeutic treatments, in order to meet the escalating clinical demands adequately, challenges still lie on stable and scalable expansion of cells.

Our team has been pinpointing at the development of a cost- effective approach for manufacturing of human stem cell. Selection of the growth medium is a crucial step for the efficacy of the expansion. Animal serum is widely utilized as a source of growth factors ingrowth medium [11,12]. However, the composition of animal serum is inconstant and unstable, and it is necessary to be tested on their suitability before use, and that would raise the operating costs tremendously $[11,12]$. Besides, the uncertain composition of serum possesses risks of transmission of infectious agents or contaminant of bacteria, fungi or virus. In view of the abovementioned drawbacks of serum containing growth medium, our team have been devoted in establishing a novel cell culture medium under serum-free conditions resulting in better consistency, lower operation cost and free-of- infection [11,12].

Human basic fibroblast growth factor (FGF2) is a well-known growth

*Address for Correspondence: Dr. Keith W.Y. Kwong, Research Department, DreamTec Research Limited, Kowloon, Hong Kong, P.R. China, E-mail: Keithkwong@dreamtec.hk

Copyright: (c) 2020 Sung J, et al. This is an open-access article distributed under the terms of the Creative Commons Attribution License, which permits unrestricted use, distribution, and reproduction in any medium, provided the original author and source are credited.

Received 16 June 2020; Accepted 22 June 2020; Published 27 July 2020 factor which is capable of replicating various types of stem cells from different sources [13-18]. Addition of FGF2 in cell culture medium efficiently promotes the proliferation, self-renewal and polypotencies of the human embryonic stem cells under feeder-free medium $[19,20]$.

In view of the authenticity and high bioactivity of FGF2 produced by our recent developed 293T expression system, we have attempted to make use of the FGF2 to formulate a cost-effective growth medium for hMSCs cultivation [15].

In this communication, we hereby report our success in employing a finetuned growth medium containing $100 \mathrm{ng} / \mathrm{mL}$ FGF2 for hMSCs expansion. The refined-hMSCs expansion protocol was shown to attain $0.8 \times 10^{5} \mathrm{hMSCs}$ cells per ml within 96 hours and confirmed to maintain $99 \%$ cell viability and hMSCs identity.

\section{Materials and Methods}

\section{Cell Culture and Transfection}

Human mesenchymal stem cells extracted from bone marrow were purchased from Merck-Millipore. Cells were either cultured in Human Mesenchymal-XF Expansion Medium or in conditioned, xeno-free medium with Dulbecco's Modified Eagle's Medium (DMEM) (Life Technologies, CA, USA) supplemented with $2 \%$ human platelet lysate, $1 \%$ Glutamax (Life Technologies, CA, USA), 1\% Penicillin-Streptomycin (Life Technologies, CA, USA) and different concentration of FGF2 at humidified incubator maintained in $37^{\circ} \mathrm{C}$ and $5 \% \mathrm{CO}_{2}$. Cells were plated at density of 5,000 cells per $\mathrm{cm}^{2}$ when $80 \%$ confluency is reached. All culture plates were coated with $0.1 \%$ gelatin solution for 30 minutes at room temperature before plating. Cell media were changed in daily basis.

\section{hMSC Proliferation Rates}

hMSCs were plated on 96 well plates and cultured in Human MesenchymalXF Expansion Medium or in conditioned, xeno-free media. The viabilities of the cells were determined by adding MTT to a final concentration of $1 \mathrm{mg} / \mathrm{mL}$ and incubated at $37^{\circ} \mathrm{C}$ for 6 hours [21]. The medium was then replaced with DMSO and the absorbance was measured in micro-plate reader at $540 \mathrm{~nm}$. 
To measure the proliferation of hMSCs, cells were cultured in different media for 2, 3, and 4 days before treating with trypsin. The trypsinized cells were then stained with trypan blue and the viable cells were counted using Thermo Fisher Countess II Automated Cell counter.

\section{Immunocytochemistry}

Cells were plated onto gelatin-coated coverslip for imaging. The cells were washed with ice-cold PBS twice, and then fixed in 4\% paraformaldehyde for $30 \mathrm{~min}$ at room temperature. The cells were subsequently blocked in $4 \%$ normal donkey serum and incubated with mouse monoclonal CD44 (1:500, CBL154), THY-1 (1:500, CBL415), STRO-1 (1:500, MAB4315), CD146 (1:500, MAB16985), CD14 (1:500, MAB1219) or CD19 (1:500, MAB1794) for overnight at $4^{\circ} \mathrm{C}$.

After washing with PBS thrice, the cells were stained with goat Alexa Fluor 647-conjugated anti-mouse antibody (1:1000, Invitrogen) and counterstained with DAPI for 1 hour at room temperature. The cells were then washed with PBS thrice before mounting on glass slides with ProLong Antifade Mountants (Thermo Fisher) forviewing.

\section{Morphological analysis}

Phase-contrast images were taken with a Nikon Eclipse Ti Inverted microscope. Images taken were analyzed with ImageJ software as previously described. Briefly, the cell's area and Feret's diameter were measured with the plug-in 'measure and label'. The hMSCswere plated and allowed to grow for 3 days before measurement. 100 cells from each culture media were measured and the experiments were performed triplicate independently. The data were then plotted on adot plot displaying area against maximum cellulardiameter.

\section{Fluorescence Activated Cell Sorting (FACS)}

Cells in $100 \mathrm{~mm}$ culture dish were trypsinized and washed with ice-cold PBS thrice. The cells were then fixed with ice-cold ethanol for 30 min at $4^{\circ} \mathrm{C}$. After fixation, the cells were incubated in 1\% BSA in PBS before blocking in 4\% normal donkey serum for $30 \mathrm{~min}$. Cells were then stained with the same primary antibodies in the immunocytochemistry section. After washing, the cells were stained with goat Alexa Fluor 488-conjugated anti-mouse antibody (Invitrogen) for $30 \mathrm{~min}$ at room temperature. The cells were then consequently washed and resuspended in PBS and analyzed on a BD FACSAria III (BD Bioscience).

\section{mRNA Quantification}

Total RNAs from hMSCs monolayer were extracted using RNAzol reagent (Molecular Research Center) according to manufacturer's instruction. The yields of RNA were quantified with Nanodrop (Thermo Fisher). 100 ug of RNA was reverse transcribed with GoScript Reverse Transcriptase (Promega) using oligo (dT) 15 primer according to manufacturer's instruction. The mRNA levels of the target genes were quantified with LightCycler 480 qPCR machine (Roche) with LightCycler 480 SYBR Green I master-mix according to manufacturer's instruction. Gene expressions were normalized against $18 \mathrm{~S}$ rRNA and calculated with $2^{\text {ict }}$. All samples were running as triplicates. The mean gene expressions were calculated within 3 independent experiments.

\section{Results}

\section{Optimization of FGF2 concentration in xeno-free medium}

Previous reports have shown that the addition of fibroblast growth factor 2 (FGF2) inhibited the differentiation of human mesenchymal stem cells (hMSCs) resulting in maintaining intact differentiability [22]. According to manufacturer's instruction, the hMSCs are grown optimally in the commercial Human Mesenchymal XF expansion medium containing human serum, with the addition of $8 \mathrm{ng} / \mathrm{mL}$ FGF2 to maintain the highest proliferation rate. The present study intended to decrease the cost of culturing hMSCs by conditioning concentration of FGF2 in DMEM to replace the use of proprietary medium. Hence, we formulated a xeno-free recipe of conditioned medium with DMEM supplied with $2 \%$ human platelet lysate, $1 \%$ Glutamax, $1 \%$ penicillin/ streptomycin and $0,8,20,50,100,150,200$, and $500 \mathrm{ng} / \mathrm{mL}$ of FGF2 for 50 passages. The cell viabilities were measured by MTT assay in day- course. Compared with the commercial XF expansion medium, our conditioned medium indeed lowered the proliferation rate of hMSCs when the FGF2 concentration was low (Figure 1A). However, starting from $50 \mathrm{ng} / \mathrm{mLFGF} 2$ in conditioned medium, the cultured cells showed a comparable proliferation rate to the commercial medium. Maximum hMSCs proliferation rate was shown when the conditioned medium was supplied with $100 \mathrm{ng} / \mathrm{mL}$ FGF2, while conditioned media with $150 \mathrm{ng} / \mathrm{mL}$ or more FGF2 showed no obvious effect on cell proliferation (Figure 1A). We repeated the cell proliferation assay by counting viable cells at different days of culture at passage number 10 , similar results showing the FGF2 concentration at $100 \mathrm{ng} / \mathrm{mL}$ provided adequate growth stimulation (Figure 1B). Viable hMSCs cultured in conditioned medium with lower FGF2 concentration $0,8,20 \mathrm{ng} / \mathrm{mL}$ could only reach $6,000,6,200$, and 9,500 cells per $\mathrm{cm}^{2}$ respectively (Figure 1B). The hMSCs grown in both commercial medium and conditioned medium with $50 \mathrm{ng} / \mathrm{mL}$ FGF2 took approximately 96 hours to reach $0.7 \times 10^{5}$ viable cells per $\mathrm{cm}^{2}$. Consequently, the proliferation rate was further enhanced that only 96 hours was taken to reach $0.8 \times 10^{5}$ viable hMSCs per $\mathrm{cm}^{2}$, with more than $95 \%$ cell viability, when a final concentration of $100 \mathrm{ng} / \mathrm{mL}$ FGF2 was provided in the conditioned medium.

\section{Validation of hMSCs with microscopy}

Although the conditioned medium with a high concentration of FGF2 supports the growth of hMSCs, the validity of stem cell markers is still to be confirmed. We maintained hMSCs until passage number 10 for 4 days in commercial XF expansion medium, and conditioned medium with 0 or $100 \mathrm{ng} /$ $\mathrm{mL}$ FGF2 supplements and the cells were observed under a phase contrast microscope. The cells cultured in both the commercial XF expansion medium and conditioned medium with $100 \mathrm{ng} / \mathrm{mL}$ FGF2 have displayed a spiral and pointy morphology (Figures 2A and $2 \mathrm{C}$ ). While the cells cultured in conditioned media without FGF2 supplements showed more flattened, epithelial type morphologies (Figure 2B). To further characterize the morphologies of hMSCs in different culture media, 100 cells were randomly picked from each culture medium and measured the cellular area and maximum cellular diameter. Results were shown on a dot-plot graph (Figure 2D). Over $90 \%$ of the cells cultured in conditioned medium with $100 \mathrm{ng} / \mathrm{mL}$ FGF2 displayed a $<8000$ $\mu \mathrm{m}^{2}$ area and $\mathrm{a}<250 \mu \mathrm{m}$ maximum diameter, which is smaller than the cells cultured with conditioned medium with $0 \mathrm{ng} / \mathrm{mL}$ FGF2 (Figure 2B). hMSCs cultured in different media were subsequently stained with mesenchymal stem cell surface marker CD44 and epithelial marker CD146. The cells cultured in both the commercial XF expansion medium and conditioned medium with 100 ng/mL FGF2 were all stained positive with mesenchymal stem cell markers CD44, and negative for epithelial marker CD146 (Figures $3 \mathrm{~A}$ and 3B). While, the cells cultured without FGF2 supplement were stained negative for mesenchymal stem cell marker (Figure $3 A$ ) and positive for non-MSC marker (Figure 3B).

\section{Validation of hMSCs with FACS}

To further validate the population of multipotent stem cells in different culture media, fluorescence assisted cell sorting (FACS) was utilized for the measurement of stem cell markers in each fraction of the hMSCs cultured in the commercial XF expansion medium and conditioned medium. Consistent with the immunocytochemistry data, at least $90 \%$ of cells cultured in the commercial XF expansion medium and conditioned medium with 100 , and 500 ng/mL FGF2 were counted positive for stem cell markers THY-1, and STRO-1 (Figures 4A and 4B). The cells were also stained and found negative for epithelial marker CD146 (Figure 4C). While the cell grown in conditioned media without FGF2 supplements were found to be negative with mesenchymal stem cell markers and positive for non-MSC marker (Figure 4).

\section{Validation of hMSCs with qPCR and Western Blotting}

To eliminate the possible artifacts due to antibody staining, we further validate the population of multipotent stem cells in different culture media utilizing quantitative polymerase chain reaction (qPCR). We employed target specific probes designed with a high specificity to the positive stem cell markers CD44, THY-1 and STRO-1 (Figure 5A). The mRNA expression levels of designated markers were quantified, which further confirmed the hMSCs 
A

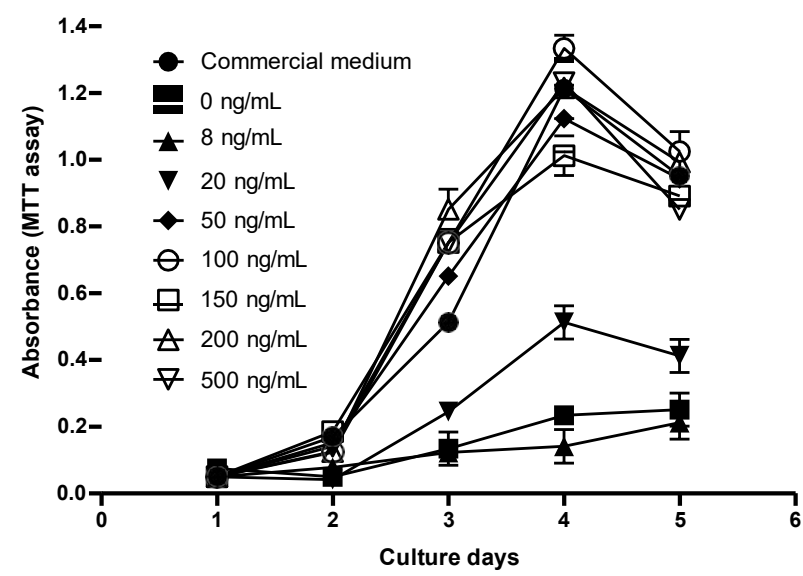

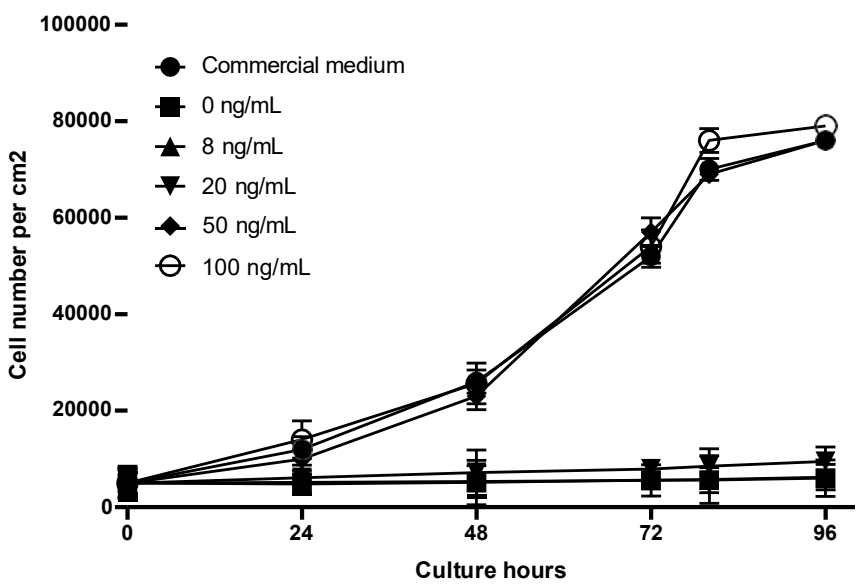

Figure 1: Proliferation of hMSC in different media. (A) hMSCs were grown at commercial Human Mesenchymal XF expansion medium with 8 ng/mL FGF2, or conditioned medium containing DMEM supplied with $2 \%$ human platelet lysate, 1\% Glutamax, 1\% penicillin/streptomycin and 0, 8, 20, 50, 100, 150, 200, and 500 ng/mL of FGF2 for 20 passages. The proliferation rates were measured by MTT assay. (B) At passage number 10,5,000 cells/cm2 hMSCs were plated at 6 well-plates and cultured in commercial Human Mesenchymal XF expansion medium with $8 \mathrm{ng} / \mathrm{mL}$ FGF2, or conditioned medium containing DMEM supplied with $2 \%$ human platelet lysate, $1 \%$ Glutamax, $1 \%$ penicillin/streptomycin and $0,8,20$, 50 and $100 \mathrm{ng} / \mathrm{mL}$ of FGF2. At the designated time points the cells were harvested and viable cells were counted.

A

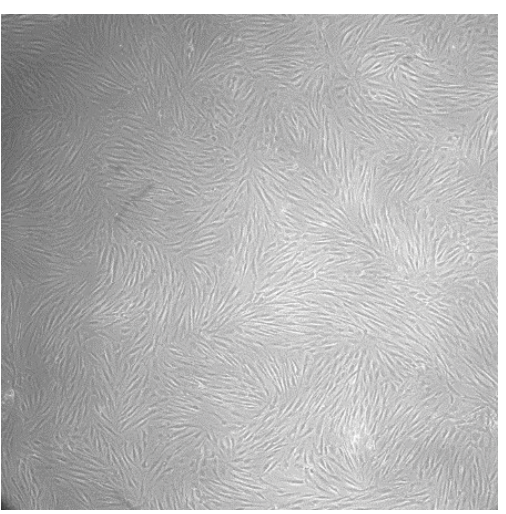

D

Commercial medium

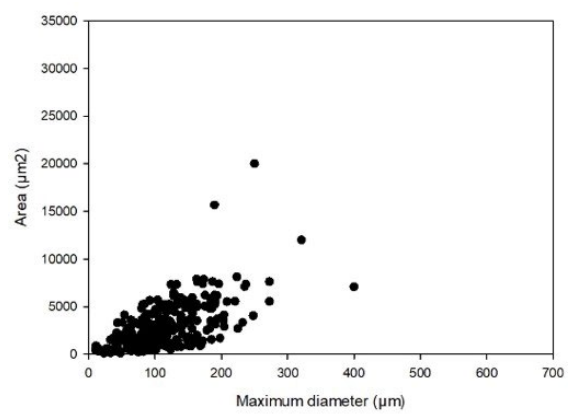

B

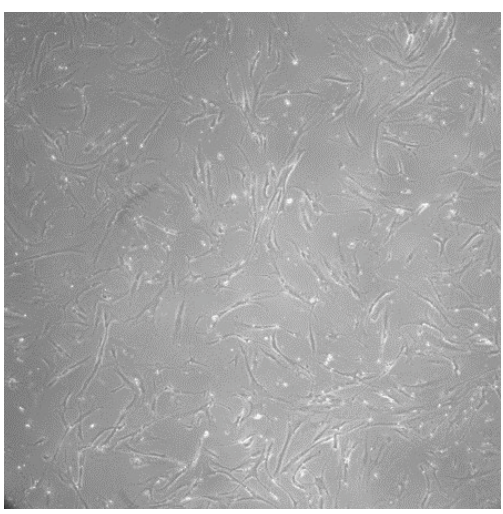

Conditioned medium + $0 \mathrm{ng} / \mathrm{mL}$ FGF2

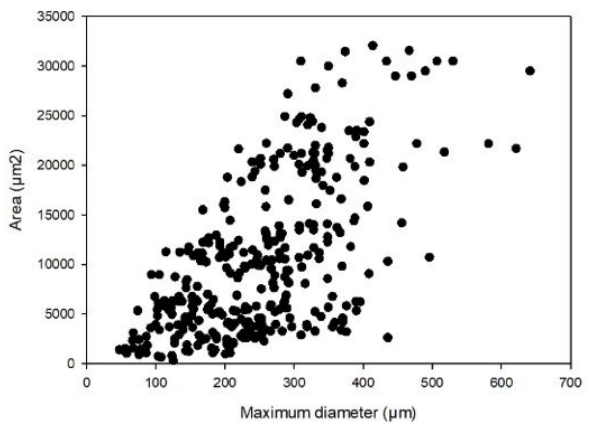

C

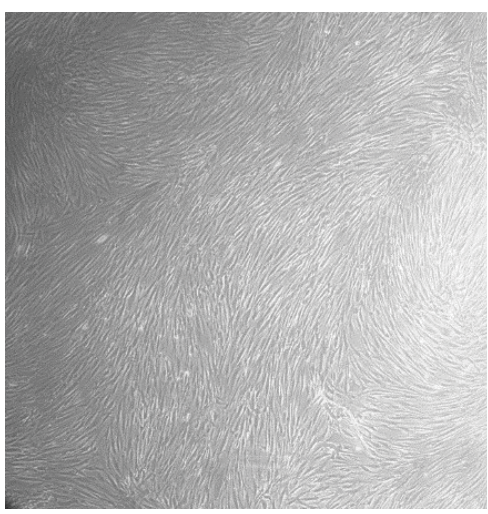

Conditioned medium + $100 \mathrm{ng} / \mathrm{mL}$ FGF2

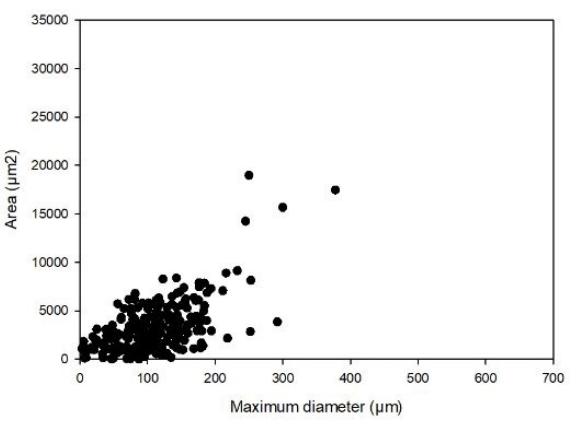

Figure 2: Validation of hMSC with microscopy (A) Wide-field images of hMSCs grown at commercial Human Mesenchymal XF expansion medium with 8 ng/mL FGF2, conditioned medium with(B) 0 and (C) $100 \mathrm{ng} / \mathrm{mL}$ of FGF2 respectively. (D) Dot blots presenting the morphological analysis on hMCSs grown in different media.

cultured in the commercial XF expansion medium and conditioned medium with $100 \mathrm{ng} / \mathrm{mL}$ FGF2 have shown similar expression levels for those stem cells markers. The cells showed no mRNA expression for epithelial marker CD146, and hematopoietic stem cells markers CD14 and CD19 (Figure $5 B$ ). While the cell cultured in media without FGF2 supplements showed no detectable mRNA level with mesenchymal stem cell markers (Figure $5 A$ ). In contrast, positive mRNA level for epithelial marker CD146 was shown (Figure $5 B$ ). None of cells expressed hematopoietic stem cell markers CD14 and CD19 (Figure5B).

To confirm the result from qPCR, Western blot was employed to check the expression level of stem cell markers CD44 and THY-1. Antibodies that are specific to the stem cell markers were employed. Consistent with the previous results, the hMSCs cultured in the commercial XF expansion medium and conditioned medium with $100 \mathrm{ng} / \mathrm{mL}$ FGF2 showed positive for the stem cells markers CD44 and THY-1, while it showed negative for epithelial marker CD146. Moreover, the cells cultured in media without FGF2 supplementswere negative for mesenchymal stem cell markers and positive for non-MSC markers (Figure 5C). 


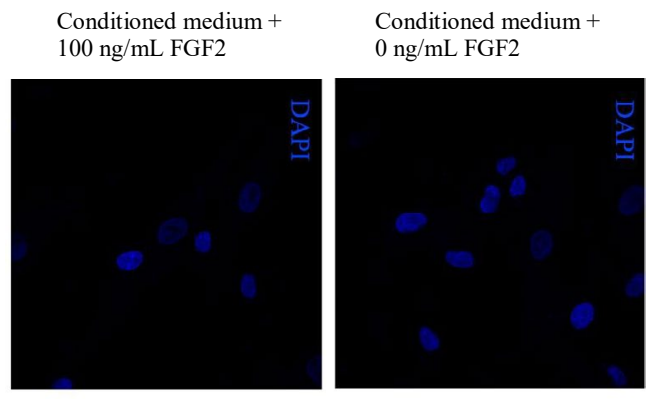

\section{Commercial medium}
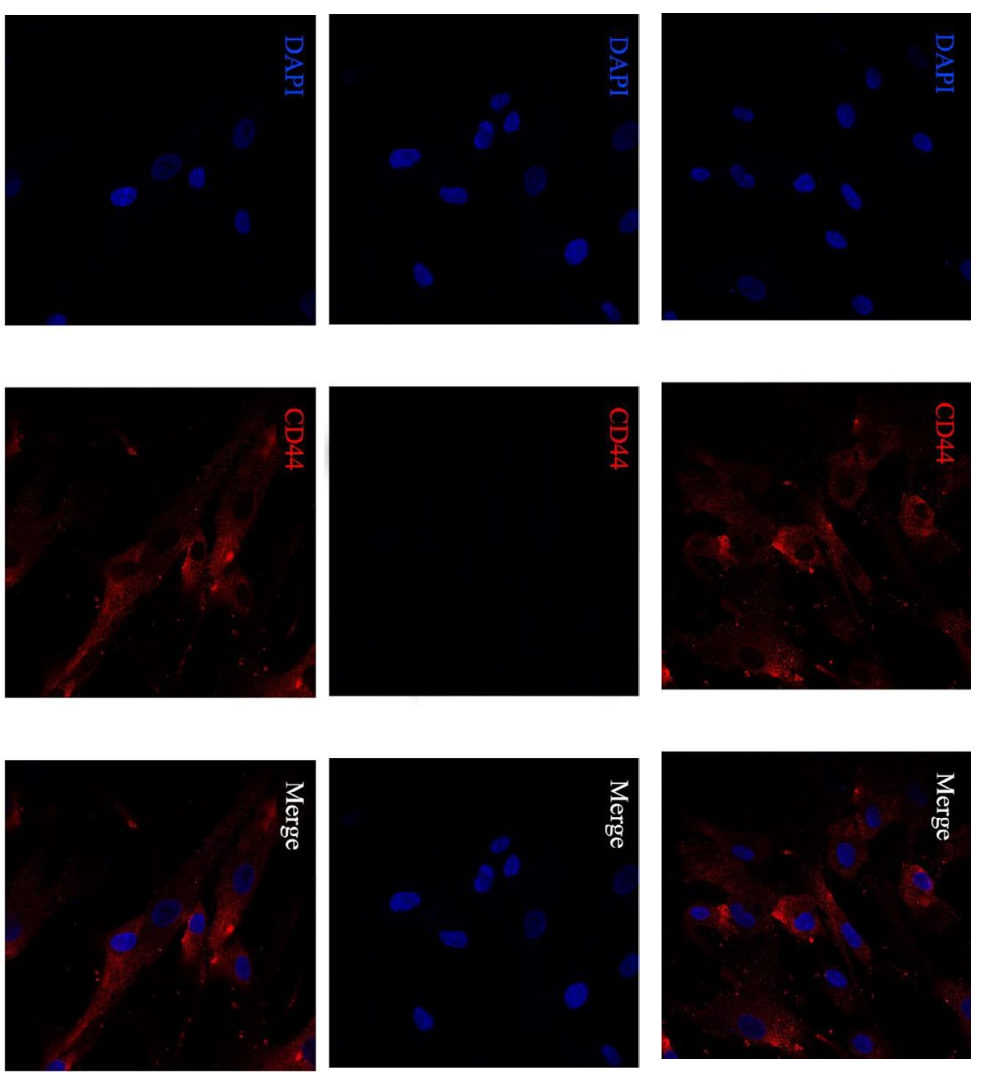

Conditioned medium + $100 \mathrm{ng} / \mathrm{mL}$ FGF2

Conditioned medium + $0 \mathrm{ng} / \mathrm{mL}$ FGF2

Commercial medium
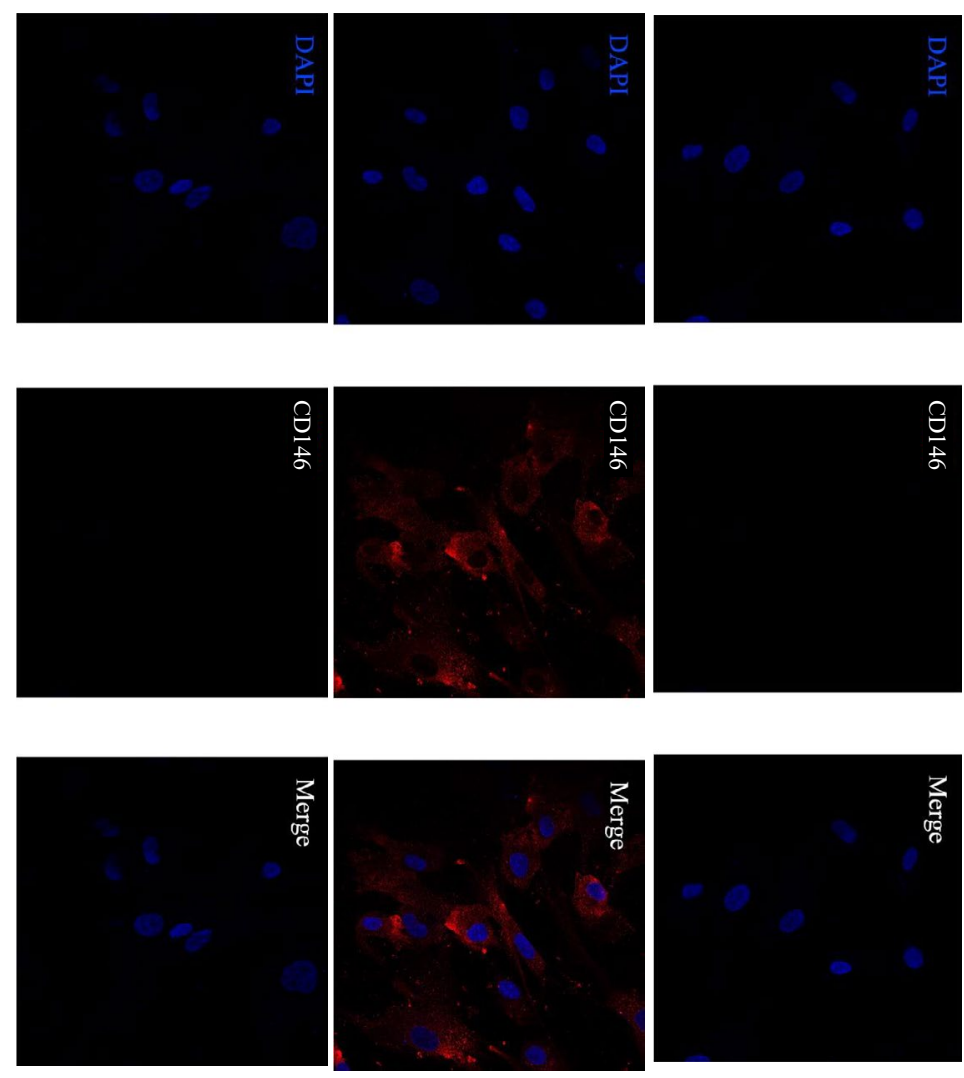

Figure 3: Validation of hMSC with microscopy. Fluorescent images of hMSC cultured in commercial Human Mesenchymal XF expansion medium with $8 \mathrm{ng} / \mathrm{mL}$ FGF2, conditioned medium with 0 and $100 \mathrm{ng} / \mathrm{mL}$ FGF2. Cells were stained with (A) MSC marker CD44, (B) epithelial marker CD146 and counterstained with DAPI. 


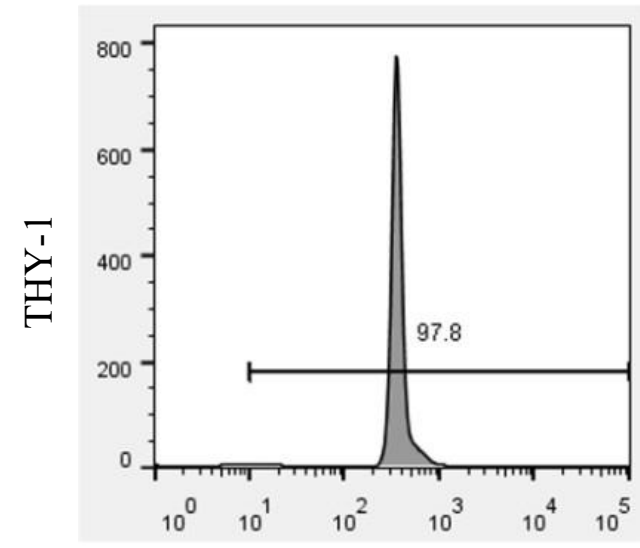

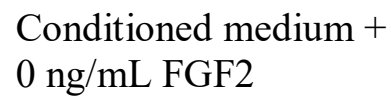

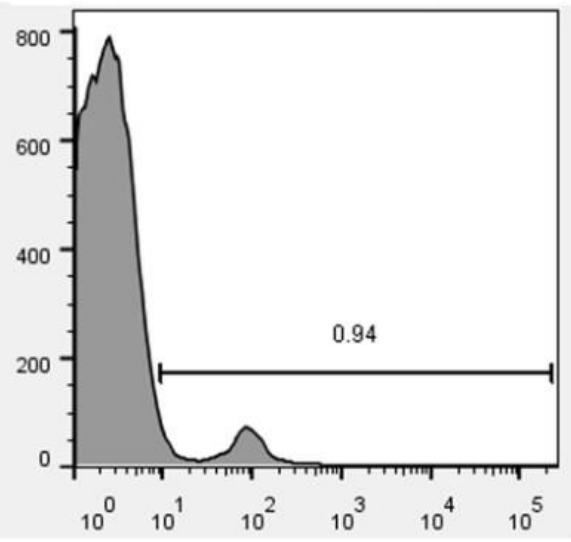

Conditioned medium + $100 \mathrm{ng} / \mathrm{mL}$ FGF2
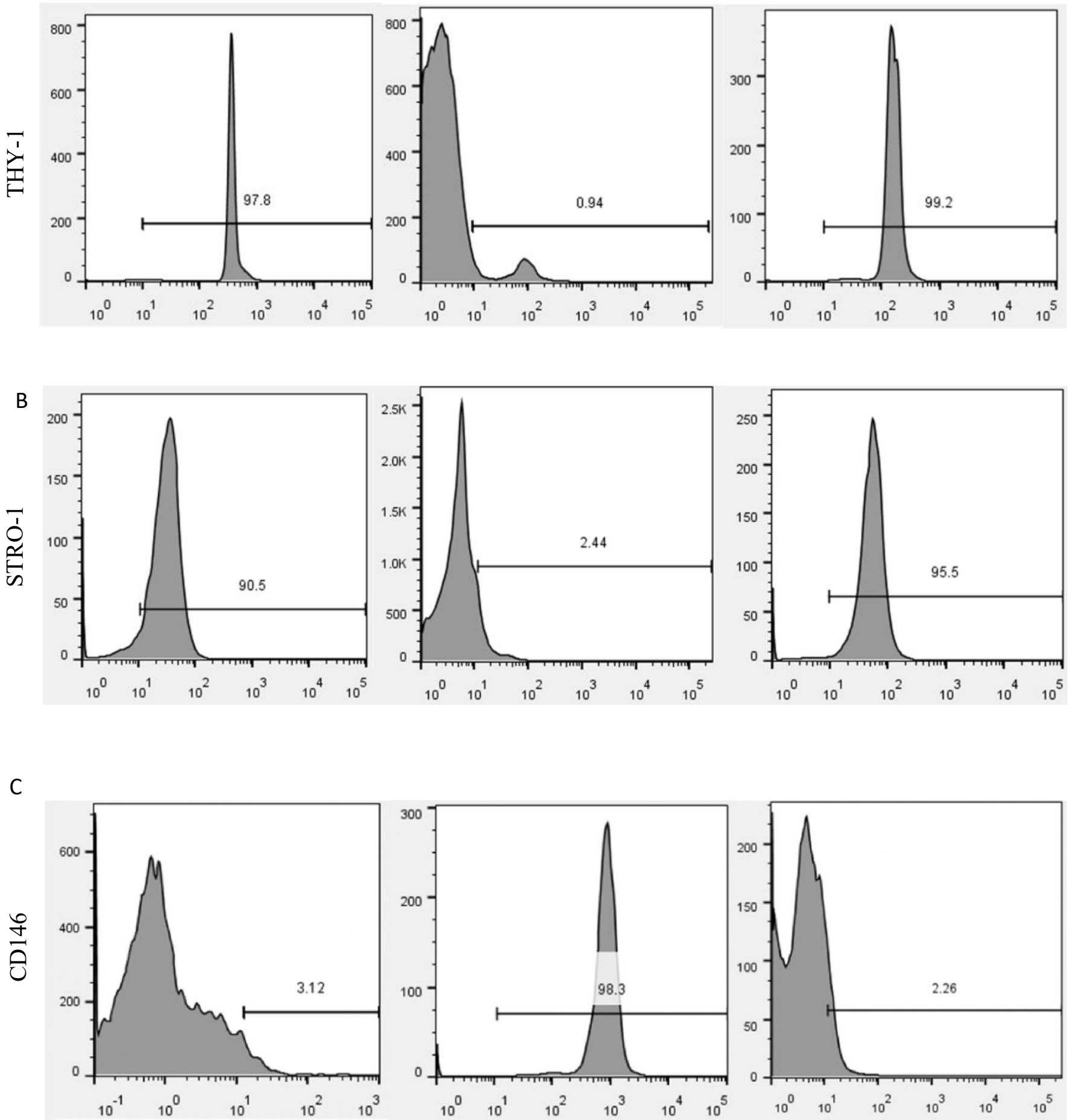

Figure 4: Validation of hMSC with FACS. Represented histogram of hMSCs grown at commercial Human Mesenchymal XF expansion medium with 8 ng/mL FGF2, conditioned medium with 0 and $100 \mathrm{ng} / \mathrm{mL}$ FGF2 stained with MSC markers (A) THY-1 and (B) STRO-1 and (C) epithelial marker CD146.

Despite the merits of stem cell in cell therapy, the applications of stem cell have so far been limited to the high production cost that requires expensive commercial growth media, such as XF Medium, Essential medium etc $[3,4,8,10,23,24]$. Although the use of serum could reduce the cost, the unknown variables, such as existence of virus, allergens, could be problematic when entering into clinical trials $[11,12]$. In order to promote the transition of stem cell from basic research to clinical applications, the development of cost-effective xeno-free media for robust expansion of human stem is absolute important.

Our group has been interested in the development and applications of regenerative medicine such as human stem cell and growth factors [14]. Human basic fibroblast growth factor (FGF2) has been efficiently expressed in human embryonic kidney 293T cell. Due to the high yield of production of FGF2 in 293T cells, we have been exploring the use of FGF2 to develop an efficient xeno-free conditioned medium for stem cell expansion. To test the ability of the FGF2 to support hMSCs culture, hMSCs were cultured in commercial XF medium and conditioned medium supplemented with various concentration of FGF2.

Our data from cell migration assay confirmed the essential role of FGF2 in 
A

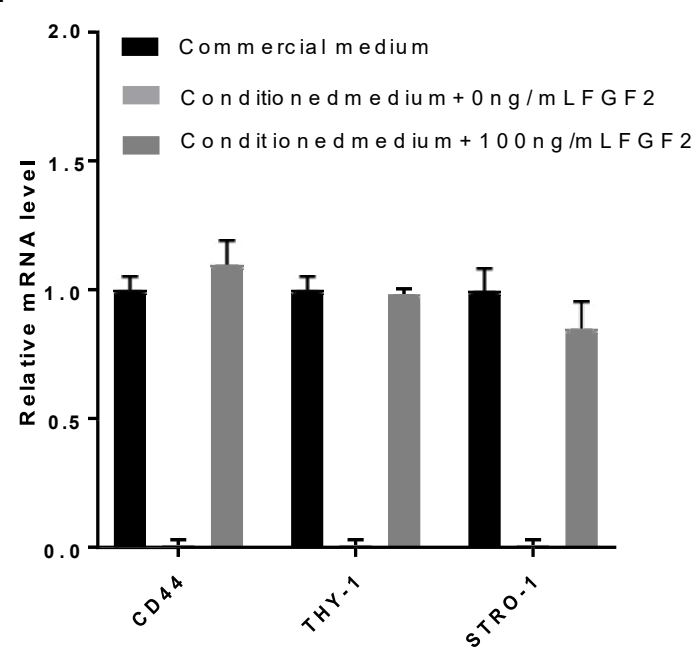

C

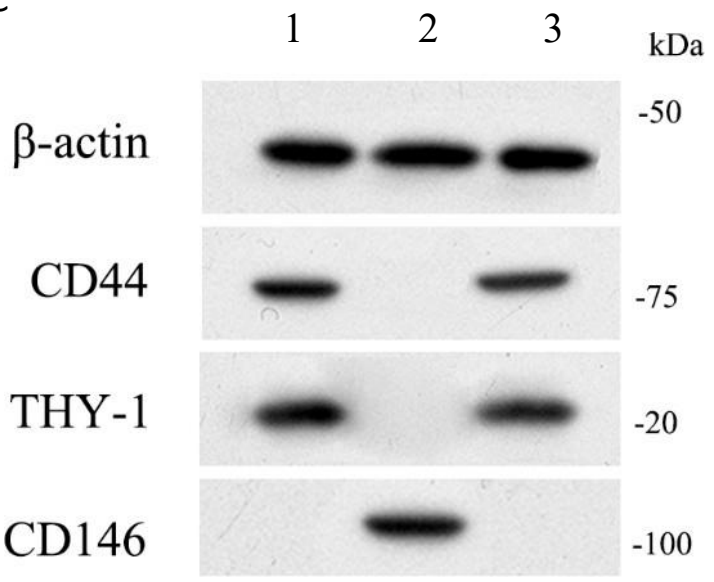

B

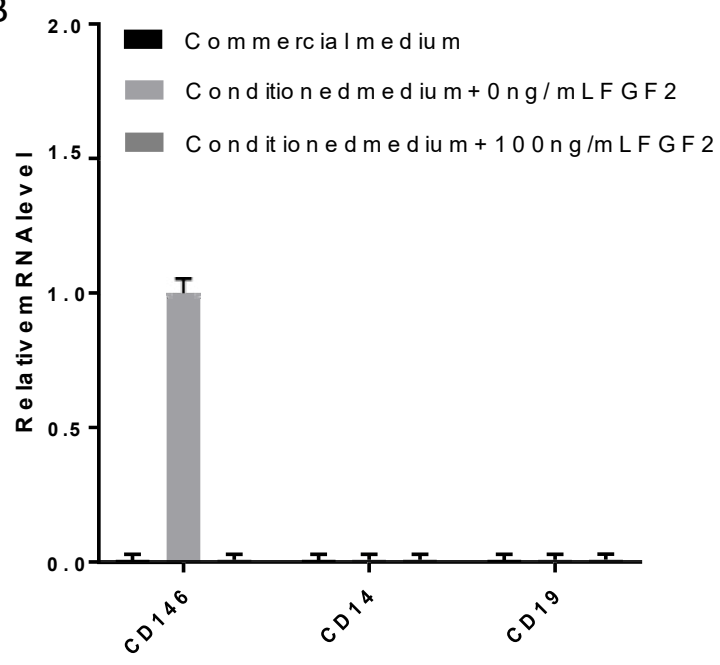

Figure 5: Validation of hMSC with qPCR and Western Blotting. mRNA from hMSCs grown at commercial Human Mesenchymal XF expansion medium with 8 ng/mL FGF2, conditioned medium with 0 and $100 \mathrm{ng} / \mathrm{mL}$ FGF2 were extracted and measured by qPCR. Expression level of (A) MSC markers CD44, THY-1 and STRO-1, (B) epithelial marker CD146, and hematopoietic stem cells markers CD14 and CD19. (C) Western Blot of the hMSCs grown at different media probed with antibodies against CD44, THY-1, and CD146. Lane 1: commercial medium, Lane 2: conditioned medium with $0 \mathrm{ng} / \mathrm{mL}$ FGF2, Lane 3: conditioned medium with $100 \mathrm{ng} / \mathrm{mL}$ FGF2

supporting hMSCs growth. Growth medium without the supplement of FGF2 is insufficient to maintain the growth of undifferentiated hMSCs (Figure 1). In our current hMSCs approach, the supplement of $100 \mathrm{ng} / \mathrm{mL}$ FGF2 resulted in an astounding yield of $0.8 \times 10^{5} \mathrm{hMSC}$ per $\mathrm{cm}^{2}$ within 96 hours in culture plate.

It is noteworthy that the morphology of cells grown in conditioned medium with concentration of $100 \mathrm{ng} / \mathrm{mL}$ or more FGF2 was more elongated and spinalshaped compared with cells grown in medium without FGF2 (Figure 2). The cells cultured in medium with $100 \mathrm{ng} / \mathrm{mL}$ FGF2, showed a shorter maximum cellular diameter and smaller cellular area than its counterpart cultured in media without FGF supplements. Such morphology is the intrinsic standard of hMSCs to be capable of growing at much higher densities and manifesting the multipotency [25].

CD44, THY-1 and STRO- 1 are the best-known hMSCs markers [10,2527].The multi-structural glycoprotein, CD44 and THY-1, expressed on the membrane surface can trigger various cellular functions including differentiation, proliferation, cell adhesion and apoptosis. The hMSCs enriched with STRO-1 promote the differentiation of the cell into multiple mesenchymal lineages, such as bone marrow stromal cells, adipocytes, osteoblasts, fibroblasts and myoblasts [26]. The hMSCs supplemented with FGF2 were shown to be positive in putative hMSCs surface marker CD44 and negative in hematologic marker CD19 under fluorescent microscope. Results from western blot and qPCR analysis further validated the undifferentiated properties of hMSCs derived in cultures with FGF2 supplements. More excitingly, when hMSCs, grown in media supplemented with FGF2, had been passaged for 50 generations, no obvious differentiation was found and the proliferation efficiency still remained the same.

\section{Conclusion}

The combination of cell viabilities, flow cytometry, morphological and immunocytochemistry characterization indicates that the addition of FGF2 is essential for the growth of undifferentiated hMSCs. Furthermore, the use of our fine-tuned culture medium supplemented with $100 \mathrm{ng} / \mathrm{mL}$ not only can efficiently facilitate the expansion of hMSCs, but it also can maintain its multipotency of differentiation into multiple lineages. This development could be helpful in the cost- effective and scalable manufacturing of hMSCs cultures for commercial and therapeutic applications.

\section{References}

1. Barlow, Sarah, Gary Brooke, Konica Chatterjee and Gareth Price, et al. "Comparison of Human Placenta and Bone Marrow-Derived Multipotent Mesenchymal Stem Cells". Stem Cells Dev 17(2008): 1095-1108.

2. Maitra, B, E Szekely, K Gjini and MJ Laughlin, et al. "Human Mesenchymal Stem Cells Support Unrelated Donor Hematopoietic Stem Cells and Suppress T-Cell Activation". Bone Marrow Transplant 33(2004): 597-604.

3. Le Blanc, Katarina and Olle Ringdén. "Immunobiology of Human Mesenchymal 
Stem Cells and Future Use in Hematopoietic Stem Cell Transplantation". Biol Blood Marrow Transplant 11(2005): 321-334.

4. Pittenger, MF, JD Mosca and KR McIntosh. "Human Mesenchymal Stem Cells: Progenitor Cells For Cartilage, Bone, Fat and Stroma". Curr Topics Microbiol Immunol 1(2000): 3-11.

5. Gao, Guifang, Arndt F Schilling, Tomo Yonezawa and Jiang Wang, et al. "Bioactive Nanoparticles Stimulate Bone Tissue Formation in Bioprinted Three-Dimensional Scaffold and Human Mesenchymal Stem Cells". Biotechnol J 9(2014): 1304-1311.

6. Gao, Guifang, Arndt F Schilling, Karen Hubbell and Tomo Yonezawa, et al. "Improved Properties of Bone and Cartilage Tissue from 3D Inkjet- Bioprinted Human Mesenchymal Stem Cells by Simultaneous Deposition and PhotoCrosslinking in PEG-GeIMA". Biotechnol Lett 37(2015): 2349-2355.

7. Crigler, Lauren, Rebecca C Robey, Amy Asawachaicharn and Dina Gaupp, et al. "Human Mesenchymal Stem Cell Subpopulations Express a Variety of NeuroRegulatory Molecules and Promote Neuronal Cell Survival and Neuritogenesis". Exp Neurol 198(2006): 54-64.

8. Cova, Lidia, Marie-Therese Armentero, Eleonora Zennaro and Cinzia Calzarossa, et al. "Multiple Neurogenic and Neurorescue Effects of Human Mesenchymal Stem Cell after Transplantation in an Experimental Model of Parkinson's Disease". Brain Res 1311(2010): 12-27.

9. Reagan, Michaela R and David L Kaplan. "Mesenchymal Stem Cell Tumor-Homing: Detection Methods in Disease Model Systems". Stem Cells 29(2011): 920-927.

10. Ding, Dah-Ching, Yu-Hsun Chang, Woei-Cherng Shyu and Shinn-Zong Lin. "Human Umbilical Cord Mesenchymal Stem Cells: A New Era for Stem Cell Therapy". Cell Transplant 24 (2015): 339-347.

11. Hemeda, Hatim, Bernd Giebel and Wolfgang Wagner. "Evaluation of Human Platelet Lysate versus Fetal Bovine Serum for Culture Of Mesenchymal Stromal Cells". Cytotherapy 16(2014): 170-180.

12. Aldahmash, Abdullah, Mandana Haack-Sørensen, May Al-Nbaheen and Linda Harkness, et al. "Human Serum is as efficient as Fetal Bovine Serum in Supporting Proliferation and Differentiation of Human Multipotent Stromal (Mesenchymal) Stem Cells in vitro and in vivo". Stem Cell Rev Rep 7(2011): 860-868.

13. Montesano, Roberto, Jean-Dominique Vassalli, A Baird and R Guillemin, et al. "Basic Fibroblast Growth Factor Induces Angiogenesis in vitro". Proc Natl Acad Sci 83(1986): 7297-7301.

14. Wu, KC, Kwong WY, Chau JCY and Choi MC, et al. "Cost-Effective Expression of Human Bio-Identical Basic Fibroblast Growth Factor in Bacillus subtilis by Employing asp DNAe Protein Intron". J Mol Genet Med 14(2020): 6.
15. Faham, S, RE Hileman, JR Fromm, RJ Linhardt, et al. "Heparin Structure and Interactions with Basic Fibroblast Growth Factor". Science 271(1996): 1116-1120.

16. Abraham, Judith $A$, Jacqueline $L$ Whang, Annette Tumolo and Ayalew Mergia, et al. "Human Basic Fibroblast Growth Factor: Nucleotide Sequence and Genomic Organization". EMBO J 5(1986): 2523-2528.

17. McGee, Gregory S, Jeffrey M Davidson, Anne Buckley and Andreas Sommer, et al. "Recombinant Basic Fibroblast Growth Factor Accelerates Wound Healing". J Surg Res 45(1988): 145-153.

18. Murakami, S, S Takayama, K Ikezawa and Y Sltimabukuro, et al. "Regeneration of Periodontal Tissues by Basic Fibroblast Growth Factor". J Periodontal Res 34(1999): 425-430.

19. https://stemcellsjournals.onlinelibrary.wiley.com/doi/full/10.1634/ stemcells.2005-0247

20. Xu, Chunhui, Elen Rosler, Jianjie Jiang and Jane S Lebkowski, et al. "Basic Fibroblast Growth Factor Supports Undifferentiated Human Embryonic Stem Cell Growth without Conditioned Medium". Stem Cells 23(2005): 315-323.

21. Gerlier, Denis and Nicole Thomasset. "Use of MTT Colorimetric Assay to Measure Cell Activation". J Immunol Methods 94: 57-63.

22. Schmidt, Annette, Dennis Ladage, Timo Schinköthe and Ursula Klausmann, et al. "Basic Fibroblast Growth Factor Controls Migration in Human Mesenchymal Stem Cells". Stem Cells 24(2006): 1750-1758.

23. Jenkins, Michael J and Suzanne S Farid. "Human Pluripotent Stem Cell-Derived Products: Advances towards Robust, Scalable and Cost-Effective Manufacturing Strategies". Biotechnol J 10(2015): 83-95.

24. Wuchter, Patrick, Marcel Vetter, Rainer Saffrich and Anke Diehlmann, et al. "Evaluation of GMP-Compliant Culture Media for in vitro Expansion of Human Bone Marrow Mesenchymal Stromal Cells". Exp Hematol 44(2016): 508-518.

25. Haasters, Florian, Wolf Christian Prall, David Anz and Carole Bourquin, et al. "Morphological and Immunocytochemical Characteristics Indicate the Yield of Early Progenitors and Represent a Quality Control for Human Mesenchymal Stem Cell Culturing". J Anat 214(2009): 759-767.

26. Ning, Hongxiu, Guiting Lin, Tom F Lue and Ching-Shwun Lin. "Mesenchymal Stem Cell Marker Stro-1 is a 75kd Endothelial Antigen". Biochem Biophys Res Commun 413(2011): 353-357.

27. Bruder, Scott P, Andreas A Kurth, Marie Shea and Wilson C Hayes, et al. "Bone Regeneration by Implantation of Purified, Culture-Expanded Human Mesenchyma Stem Cells". J Orthop Res 16(1998): 155-162.

How to cite this article: Sung J, Wu KC, Choi MC and Ma CHY, et al. "Development of a Cost-effective Xeno-free Conditioned Medium Containing Human Basic Fibroblast Growth Factor for the Expansion of Human Mesenchymal Stem Cell." J Mol Genet Med 14 (2020): 459 doi: 10.37421/jmgm.2020.14.459 